\title{
Gouvernance De La Planification Familiale Et Realite Sociale Au Benin
}

\author{
Edgard Odjo Cledjo \\ Université d'Abomey-Calavi / DS-A / FASHS, Bénin \\ Fanès Azalou Tingbe (PhD) \\ Université d'Abomey-Calavi / DGAT/FASHS, Bénin
}

Doi:10.19044/esj.2018.v14n23p138 URL:http://dx.doi.org/10.19044/esj.2018.v14n23p138

\begin{abstract}
This research aims to analyze the role and practices of actors in the field of Family Planning in Benin. It is based on a methodological approach that takes into account the collection of data from 209 people (civil society actors, adopters, state actors, etc.), selected by reasoned choice. The data collected were analyzed and the results were analyzed according to the actors' games in a comprehensive perspective and a more holistic approach than methodological individualism. The results show that technical and financial partners are the "dominant actor" in FP in Benin, their decisions and projects are crucial for the evolution of contraceptive prevalence in Benin. The state, NGOs, adopters of FP methods and the general population constitute "relay actors". Traditional chiefdoms, religious denominations, populations, and to some extent development associations are in a position quite far from the goals of FP. On the other hand, NGOs, adopters of planning methods and technical and financial partners are at the center of the fray, and therefore at the heart of a network of several objectives. With the difficulties mentioned by the actors and the disadvantages mentioned by the adopters, family planning, in order to be accepted and become effective, must not go against the socio-cultural and economic norms that govern the conditions of reproduction.
\end{abstract}

Keywords: Benin, family planning, CSO, contraceptive methods, health.

\section{Résumé}

Cette recherche vise à analyser le rôle et les pratiques des acteurs dans le champ de la Planification Familiale au Bénin. Elle s'appuie sur une approche méthodologique qui prend en compte la collecte des données auprès de 209 personnes (acteurs de la société civile, adoptants, acteurs étatiques, etc.), sélectionnés par choix raisonné. Les données recueillies ont été dépouillés et les résultats ont été analysés suivant les jeux d'acteurs dans une 
perspective compréhensive et une approche plus holistique que l'individualisme méthodologique. Il ressort des résultats que les PTF sont l'acteur dominant de la PF au Bénin, leurs décisions et projets sont déterminants pour l'évolution de la prévalence contraceptive au Bénin. L'Etat, les ONG, les adoptants des méthodes de PF et les populations en général constituent des «acteurs relais». Les chefferies traditionnelles, les confessions religieuses, les populations et dans une certaine mesure les associations de développement sont dans une position assez éloignée des objectifs de la PF. Par contre, les ONG, les adoptants des méthodes de planification et les partenaires techniques et financiers se trouvent au centre de la mêlée, et donc au cœur d'un réseau de plusieurs objectifs. Avec les difficultés évoquées par les acteurs et les inconvénients mentionnés par les adoptants, la planification familiale, pour être acceptée et devenir efficace, ne doit pas aller à l'encontre des normes socio-culturelles et économiques qui régissent les conditions de la reproduction.

Mots-clés : Bénin, planification familiale, OSC, méthodes contraceptives, santé.

\section{Introduction}

A l'origine, la planification familiale (P.F) est considérée comme un domaine difficile à investir voire un domaine à combattre du fait de son opposition flagrante avec la mentalité béninoise pro-nataliste et en conflit avec les habitudes socio-culturelles. En effet, l'accès aux services de la planification familiale est très limité (P. Delanne et J. G. Guingnido, 2003). En dépit de son rôle dans la réduction de la mortalité maternelle et la maîtrise de la fécondité, elle ne fait pas l'objet de préoccupation majeure, et est reléguée au second plan, tant du point de vue des ressources allouées aux programmes que l'engagement des acteurs stratégiques et opérationnels en faveur des services de PF (C. Kuyu et D. Kéita, 2011).

L'intérêt et l'importance de la planification familiale a amené le Gouvernement béninois à faire voter par l'Assemblée Nationale le 3 Mars 2003, la loi N²003-03 relative à la santé sexuelle et à la reproduction qui abroge ainsi celle de 1920. Cette loi confère à toute personne « le droit d'être informé et d'utiliser la méthode de planification familiale de son choix, qui ne soit pas contraire à la loi ». Ainsi, sous la houlette des partenaires, dans le cadre de la coopération internationale, la promotion de la planification familiale a été repositionnée comme stratégie de lutte contre la pauvreté, contre la mortalité maternelle et infantile et comme une stratégie de développement. Le Ministère de la santé avec l'appui du Fonds des NationsUnies pour la population a élaboré en 2009 une stratégie nationale de repositionnement de la planification familiale. 
Au Bénin, les méthodes contraceptives se classent en deux groupes : les méthodes modernes et les méthodes traditionnelles. Les méthodes modernes comprenant la stérilisation féminine et la stérilisation masculine, la pilule, le stérilet ou DIU (Dispositif Intra Utérin), les injectables, les implants (Norplant), le condom masculin, le condom féminin, le diaphragme, les méthodes vaginales (spermicides, mousses et gelées), la Méthode de 1'Allaitement Maternel et de l'Aménorrhée (MAMA) et la pilule du lendemain (D. Barcelona et al., 1982). Les méthodes traditionnelles comprennent les méthodes dites « populaires », comme les herbes, les tisanes, les amulettes, et autres méthodes (E. Wallace et E. Goundété, 1996). Pour T. Hountondji (2012), certaines femmes recourraient à des pilules comme la Nivaquine, l'Aspirine ou le «Sédaspir », et de boissons comme la Guinness, pour ne pas concevoir. En effet, ces «médicaments» auraient des effets abortifs chez certaines femmes. Toutefois, aucune expérimentation ne corrobore cette thèse.

Les organisations de la société civile au Bénin longtemps opposées pour la plupart aux méthodes contraceptives modernes, s'invitent spontanément ou sont appelées à jouer divers rôles. Cet engouement a contribué à faire passer la prévalence contraceptive moderne de 6,2\% selon l'EDS 2006 à $8 \%$ selon l'EDS 2011. Malgré cette évolution de la prévalence contraceptive, elle reste largement en deçà de l'objectif de $15 \%$ en 2015 tel qu'il figure dans les documents stratégiques nationaux à savoir la DEPOLIPO et la stratégie nationale de repositionnement de la planification familiale. La prévalence contraceptive relativement faible du Bénin en comparaison avec la moyenne des pays en Afrique est une source d'inquiétude qui amène les dirigeants et les différents acteurs à intensifier leurs actions. C'est pour cette raison que le présent article s'est penché sur la question. Il s'agit d'analyser le jeu des acteurs de la planification familiale pour identifier les rôles, logiques et pratiques qui structurent leurs actions d'une part, et établir une hiérarchisation selon les enjeux et objectifs de la PF au Bénin d'autre part.

\section{1- Données et méthodes}

La démarche de recherche associe les approches qualitative et quantitative. La première s'explique par le fait que la recherche s'est plus focalisée sur l'étude du sens des phénomènes sociaux autour de la planification familiale au Bénin dans une perspective compréhensive. L'approche quantitative, quant à elle, relève de la nécessité d'illustrer les comportements et les interactions entre les différents acteurs de la planification familiale au Bénin; elle a conduit à l'utilisation de certaines méthodes d'analyse ayant pour base des modes de calculs mathématiques ou statistiques.

Les données utilisées sont celles collectées lors de la recherche documentaire et des enquêtes de terrain. Il s'agit des données socio-sanitaires, 
des données liées à la santé de la reproduction et la planification familiale au Bénin, des données sociodémographiques et des données sociologiques recueillies directement sur le terrain auprès des groupes cibles concernés par la recherche. Il s'agit des organisations de la société civile intervenant pour ou contre la planification familiale, des structures étatiques, des élus locaux et des bénéficiaires des services de la planification familiale.

Pour la collecte des données de terrain, deux techniques d'investigation ont été utilisées pour la collecte des informations. Il s'agit de l'observation directe et de l'entretien semi-directif. Si tous les départements du Bénin ont été effectivement visités dans le cadre de cette recherche, les départements de l'Atlantique, du Littoral et de l'Ouémé au sud et le département du Borgou au nord-est, spécifiquement les villes de Cotonou, Porto-Novo, Parakou, et Abomey-Calavi ont été les plus fréquentés du fait d'une concentration des organisations de la société civile. La taille de l'échantillon a été obtenue après l'atteinte du point de saturation de l'information. Au total, 209 personnes ont été interrogées dans les départements de l'Atlantique, du Littoral, de l'Ouémé et du Borgou. Cet effectif total est composé de 96 acteurs de la société civile et 113 adoptants des méthodes de planification familiale.

Les données collectées ont été saisies et analysées à l'ordinateur. Il a été procédé à la catégorisation, au classement puis à la triangulation des données qualitatives recueillies. Les données quantitatives ont été directement intégrées dans le logiciel MACTOR, puis illustrez avec des graphiques grâce au tableur Excel. S'agissant de l'appréciation du jeu des acteurs, le logiciel d'analyse prospective MACTOR (M. Godet, 2001) a été utilisé pour obtenir des matrices et des schémas des positionnements, de convergences/divergences des différents acteurs intervenant dans le domaine de la planification familiale au Bénin.

\section{2- Résultats}

\section{2-1- Enjeux et objectifs de la planification familiale au Bénin}

La Planification Familiale, considérée comme composante essentielle des soins de santé primaire et de santé de la reproduction, joue un rôle important dans la réduction des taux de morbidité et de mortalité maternelle et néonatale. Elle reste et demeure un moyen sûr pour mettre en adéquation la croissance démographique et la croissance économique. L'analyse de la stratégie nationale de repositionnement de la planification au Bénin et les entretiens conduits avec les différents acteurs intervenant dans le domaine permet d'identifier les enjeux et objectifs de la planification familiale au Bénin (tableau I). 
Tableau I : Enjeux et objectifs de la planification familiale au Bénin

\begin{tabular}{|c|c|c|}
\hline $\begin{array}{l}\text { Domaines } \\
\text { prioritaires }\end{array}$ & Enjeux & Objectifs associés \\
\hline \multirow[b]{2}{*}{ Communication } & \multirow[b]{2}{*}{$\begin{array}{l}\text { Renforcement de la } \\
\text { communication }\end{array}$} & $\begin{array}{l}\text { OS1- Elaborer et mettre en œuvre un } \\
\text { plan de plaidoyer en direction des } \\
\text { décideurs }\end{array}$ \\
\hline & & $\begin{array}{l}\text { OS2- Elaborer et mettre en œuvre un } \\
\text { plan intégré de mobilisation sociale et } \\
\text { de Communication pour un } \\
\text { Changement de Comportement }\end{array}$ \\
\hline \multirow{4}{*}{$\begin{array}{l}\text { Accès aux } \\
\text { services }\end{array}$} & \multirow{4}{*}{$\begin{array}{c}\text { Amélioration de l'accès aux } \\
\text { services de PF de qualité à } \\
\text { tous les niveaux }\end{array}$} & $\begin{array}{l}\text { OS3- Renforcer la capacité des } \\
\text { prestataires de soins en sécurisation des } \\
\text { produits de la Planification Familiale }\end{array}$ \\
\hline & & $\begin{array}{l}\text { OS4- Réorganiser les services de santé } \\
\text { pour une meilleure intégration de la PF }\end{array}$ \\
\hline & & $\begin{array}{l}\text { OS5- Renforcer les compétences de } \\
\text { tous les prestataires de soins }\end{array}$ \\
\hline & & $\begin{array}{l}\text { OS6- Améliorer l'accessibilité } \\
\text { financière de la PF }\end{array}$ \\
\hline \multirow{3}{*}{$\begin{array}{c}\text { Cadre } \\
\text { institutionnel }\end{array}$} & \multirow{3}{*}{$\begin{array}{c}\text { Renforcement du cadre } \\
\text { institutionnel et juridique en } \\
\text { matière de PF }\end{array}$} & $\begin{array}{lll}\text { OS7- Vulgariser et mettre en } & \text { en } \\
\text { application les lois sur la SR } & \\
\end{array}$ \\
\hline & & $\begin{array}{l}\text { OS8- Renforcer le mécanisme de } \\
\text { coordination des interventions de PF au } \\
\text { niveau national }\end{array}$ \\
\hline & & $\begin{array}{l}\text { OS9- Améliorer l'enseignement de la } \\
\text { PF dans les écoles de formation de } \\
\text { base des professionnels de santé et des } \\
\text { structures connexes }\end{array}$ \\
\hline \multirow{2}{*}{$\begin{array}{l}\text { Accessibilité } \\
\text { financière }\end{array}$} & \multirow{2}{*}{$\begin{array}{l}\text { Mobilisation des ressources } \\
\text { pour le repositionnement de } \\
\text { la PF }\end{array}$} & $\begin{array}{l}\text { OS10- Assurer le financement par le } \\
\text { budget de l'Etat de l'approvisionnement } \\
\text { en produits de PF }\end{array}$ \\
\hline & & $\begin{array}{llr}\text { OS11- Mobiliser } & \text { les } & \text { ressources } \\
\text { additionnelles } & \text { pour } & \text { le } \\
\text { repositionnement de la PF } & \\
\end{array}$ \\
\hline Suivi/évaluation & $\begin{array}{c}\text { Amélioration du } \\
\text { suivi/évaluation en matière } \\
\text { de PF }\end{array}$ & $\begin{array}{l}\text { OS12- Renforcer le mécanisme de } \\
\text { suivi/évaluation des activités de PF }\end{array}$ \\
\hline
\end{tabular}

Source : Données de terrain, février-novembre, 2015.

Dans le domaine de la communication sociale, l'enjeu est le renforcement de la communication, à travers l'élaboration et la mise en œuvre de plan de plaidoyer envers les décideurs et d'un plan intégré de mobilisation sociale et de communication pour un changement de comportement. L'amélioration de l'accès aux services de la planification familiale est un défi à relever au Bénin. Pour ce faire, il s'agira d'intervenir sur les capacités des prestataires de soins, la sécurisation des produits de PF, l'intégration de la PF dans les services de santé et l'accessibilité financière de la PF. 
Pour assurer l'enjeu du renforcement du cadre institutionnel et juridique de la planification familiale, les objectifs visent la vulgarisation et l'application des lois sur la santé de la reproduction, le renforcement du mécanisme de coordination des interventions, l'intégration de l'enseignement de la PF dans les centres de formation en santé. Au plan de la mobilisation des ressources pour le repositionnement de la PF, il s'agit d'assurer le financement par le budget de l'Etat de l'approvisionnement en produits de PF. L'amélioration du système de suivi / évaluation des activités de la PF, reste un énorme défi à relever, au regard de la faiblesse des structures et des mécanismes existants. Le suivi est impérieux car dans le domaine de la PF, on observe une multiplicité d'acteurs aux caractéristiques et intérêts parfois divergents, mais agissant pour la plupart pour l'atteinte d'un seul objectif : améliorer la prévalence contraceptive au Bénin.

\section{2-2- Structure des influences entre les acteurs de la planification familiale au Bénin}

L'analyse des influences et dépendances des acteurs de la PF permet de dégager les rapports de force et la marge de manœuvre des acteurs.

\section{2-2-1- Influences et dépendances des acteurs}

Plusieurs acteurs interagissent dans le domaine de la PF en vue de l'atteinte des objectifs. Dans le cadre de cette recherche, les différents acteurs ont été regroupés en onze catégories : l'Etat, les partenaires techniques et financiers (PTF), les organisations non gouvernementales (ONG), les confessions religieuses (Religieux), les chefferies traditionnelles (Tradition), les ordres professionnels (Ordres), les syndicats (Syndicats), les organisations de presse (Presse), les associations de développement (AD), les adoptants des méthodes de PF (Adoptants), les autres catégories de la population (Population). La structure des influences entre les acteurs permet de procéder à leur hiérarchisation, afin d'identifier les acteurs dominants et ceux qui sont situés hors du système. Elle se fait sur la base de la Matrice des Influences Directes (MID) entre acteurs (tableau II). 
Tableau II : Matrice des influences directes et indirectes (MIDI)

\begin{tabular}{|c|c|c|c|c|c|c|c|c|c|c|c|c|}
\hline MIDI & $\underset{\vec{T}}{\stackrel{\mathbb{N}}{\rightleftharpoons}}$ & 勇 & $\stackrel{O}{Z}$ & 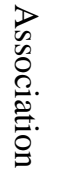 & 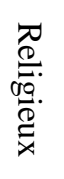 & 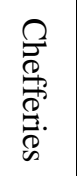 & $\begin{array}{l}\text { O } \\
\stackrel{0}{8} \\
\stackrel{8}{\infty}\end{array}$ & 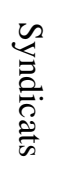 & $\begin{array}{l}\vec{D} \\
\vec{D} \\
0 \\
0\end{array}$ & 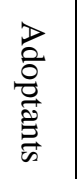 & 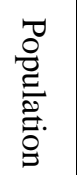 & 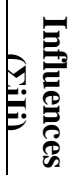 \\
\hline Etat & 17 & 12 & 17 & 11 & 12 & 13 & 8 & 11 & 11 & 21 & 20 & 136 \\
\hline PTF & 19 & 12 & 19 & 11 & 13 & 14 & 9 & 12 & 12 & 25 & 24 & 158 \\
\hline ONG & 17 & 10 & 13 & 8 & 10 & 11 & 6 & 9 & 9 & 22 & 20 & 122 \\
\hline Association & 5 & 4 & 6 & 4 & 6 & 6 & 3 & 6 & 6 & 6 & 6 & 54 \\
\hline Religieux & 10 & 9 & 11 & 9 & 8 & 9 & 5 & 8 & 8 & 14 & 12 & 95 \\
\hline Chefferies & 9 & 8 & 9 & 8 & 8 & 8 & 5 & 8 & 8 & 9 & 9 & 81 \\
\hline Ordres & 8 & 7 & 8 & 7 & 8 & 8 & 5 & 8 & 8 & 8 & 8 & 78 \\
\hline Syndicats & 7 & 6 & 8 & 6 & 8 & 8 & 5 & 8 & 8 & 8 & 8 & 72 \\
\hline Presse & 10 & 9 & 11 & 9 & 9 & 10 & 5 & 8 & 8 & 12 & 11 & 94 \\
\hline Adoptants & 14 & 10 & 16 & 10 & 12 & 13 & 8 & 11 & 11 & 19 & 19 & 124 \\
\hline Population & 14 & 11 & 16 & 9 & 10 & 11 & 6 & 9 & 9 & 20 & 18 & 115 \\
\hline Dépendances ( $\mathrm{iiDi})$ & 113 & 86 & 121 & 88 & 96 & 103 & 60 & 90 & 90 & 145 & 137 & 112 \\
\hline
\end{tabular}

Source : Données de terrain, février-novembre, 2015.

L'analyse du tableau II permet de retenir que les partenaires techniques et financiers (PTF) sont les acteurs les plus influents. Ils totalisent la somme des influences la plus élevée. L'Etat vient en deuxième position des acteurs les plus influents, ensuite les adoptants et les ONG. Tout en étant parmi les acteurs les plus influents de la planification familiale au Bénin, les adoptants apparaissent également comme les plus soumis à l'influence des autres acteurs. Ils sont suivis de la population en général (regroupant ceux qui n'adoptent aucune méthode de PF), des ONG et de l'Etat. Sur l'ensemble, on remarque que quatre des cinq acteurs les plus influents du domaine de la PF au Bénin sont également parmi les plus soumis aux influences des autres acteurs. L'analyse des rapports de force directs et indirects, ainsi que des boucles de rétroaction devraient permettre de mieux appréhender les interrelations entre ces acteurs.

Les ordres professionnels, les associations de développement, les syndicats, la presse et les confessions religieuses sont parmi les acteurs les moins influents, mais également les moins dépendants des autres acteurs. Ainsi, sur la base de cette matrice, on peut observer le positionnement des acteurs dans le domaine de la planification familiale au Bénin (Figure 1). Ce plan est calculé à partir de la matrice MIDI (Ii et Di). Il permet de visualiser en abscisses la dépendance et en ordonnées l'influence des acteurs entre eux (N. Bassaler, 2004). 


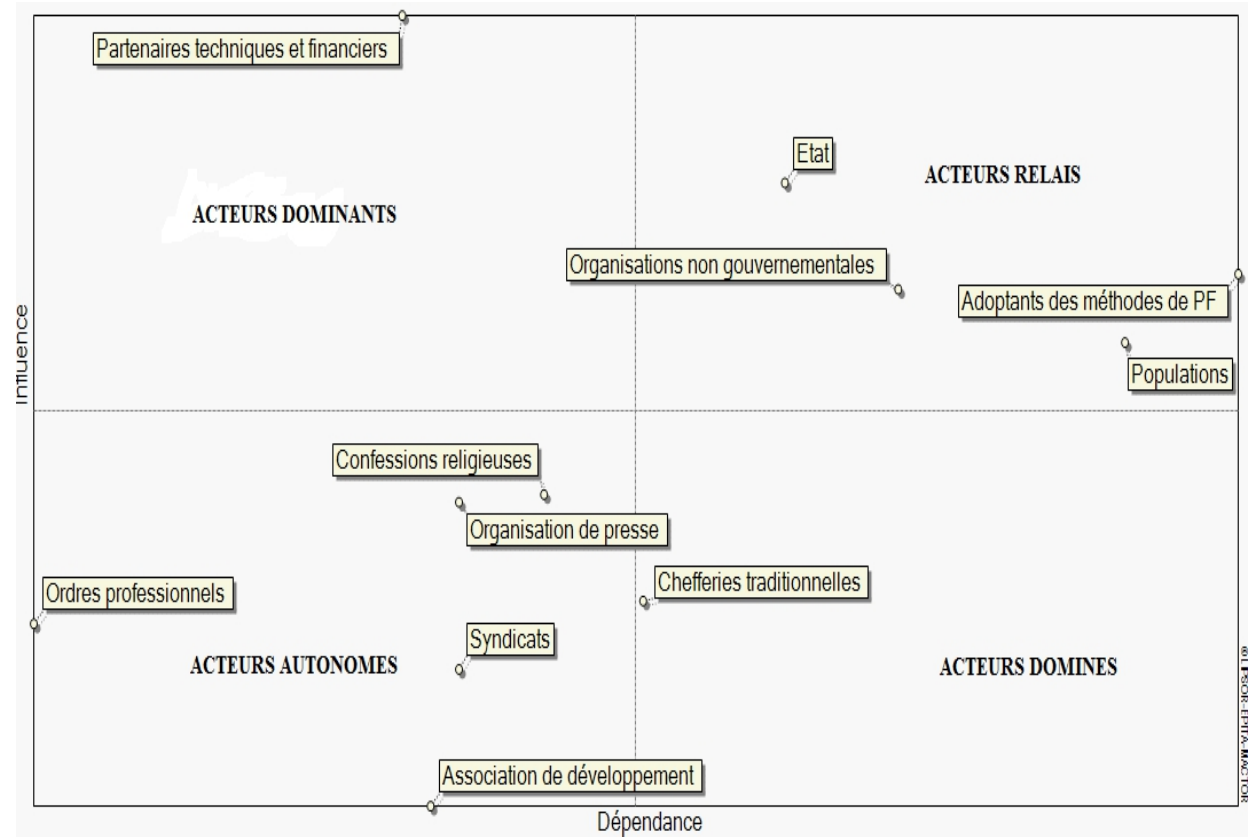

Figure 1 : Plan des influences et dépendances entre les acteurs de la planification familiale au Bénin

Source : Données de terrain, février-novembre, 2015.

L'analyse de la figure 1 montre que les PTF sont les «acteurs dominants » de la planification familiale au Bénin. Ils disposent d'une influence forte sur les autres acteurs, sans être eux-mêmes fortement influencés. Ils sont les principaux acteurs à l'origine des différentes orientations prises par la politique nationale dans le domaine de la planification familiale. Ils interviennent à tous les niveaux de la chaîne ; depuis la prise de décision, jusqu'à la cession des produits aux adoptants, en passant par le financement des projets et programmes. Les PTF à travers plusieurs organismes internationaux constituent les acteurs initiateurs d'évolutions déterminantes pour la planification au Bénin. La recherche d'alliances et la minimisation des conflits doivent être recherchées avec cet acteur influent.

Dans le domaine de la régulation des naissances, l'Etat, les ONG, les adoptants des méthodes de $\mathrm{PF}$ et les populations en général constituent des « acteurs relais ». Ce groupe d'acteurs est essentiel dans la définition et la mise en œuvre de la politique nationale de planification familiale. Les « acteurs relais » sont les plus impliqués dans la structure des influences. Si l'Etat, à travers les structures de l'exécutif, est seul à disposer de la compétence pour l'initiative de la politique, ce sont les ONG qui la mettent en application et constitue l'interface entre les structures étatiques, les PTF et les bénéficiaires des méthodes de PF. Ces « acteurs relais » déterminent le degré d'ouverture 
du jeu (N. Bassaler, 2004). En effet, si les projets et les objectifs de ce groupe relais ne se réalisent pas, ou si leurs opinions ne sont pas prises en compte, la dynamique de la planification familiale ne saurait évoluée. Ainsi, c'est autour de l'Etat, des ONG, des adoptants de méthodes de PF et les populations que devraient s'organiser les jeux de négociation entre acteurs aux conséquences importantes.

Les chefferies traditionnelles sont très sensibles aux évolutions du domaine. Ces acteurs se retrouvent dans le cadran des « acteurs dominés ». Ils sont plus dépendants qu'influents et ne peuvent construire des projets structurants dans le domaine de la PF. Ils sont des acteurs plutôt menacés et obligés de s'adapter sans cesse. Très proches du groupe des acteurs relais, de par leur positionnement dans le plan, il parait peu prudent pour ces derniers de recourir à ces seuls acteurs en terme d'appui ou de jeux d'alliances.

Les confessions religieuses, les ordres professionnels, les syndicats, les organisations de presse et les associations de développement apparaissent comme des acteurs faiblement connectés au domaine de la planification familiale au Bénin. Ils forment le groupe des «acteurs autonomes ». Ce positionnement est révélateur du niveau d'intégration psychosociale de la réalité de la PF au Bénin. Ces acteurs ne se sentent pas concernés par cette problématique et n'appréhendent pas réellement leur rôle et contribution dans les méthodes modernes de régulation des naissances. Certains leaders des confessions religieuses n'hésitent d'ailleurs pas à initier des contre-campagnes et afficher clairement leur opposition à ces méthodes.

\section{2-2-2- Rapports de force directs et indirects entre acteurs de la planification familiale}

Le rapport de force d'un acteur (Ri) est un indicateur qui permet d'apprécier son poids relatif dans la régulation du jeu (N. Bassaler, 2004). Plus il est élevé, plus l'acteur pèse dans le jeu ; plus il est faible, moins l'acteur est en position de défendre ses intérêts. La figure 2 illustre les rapports de force directs et indirects entre les acteurs de la planification familiale au Bénin. 


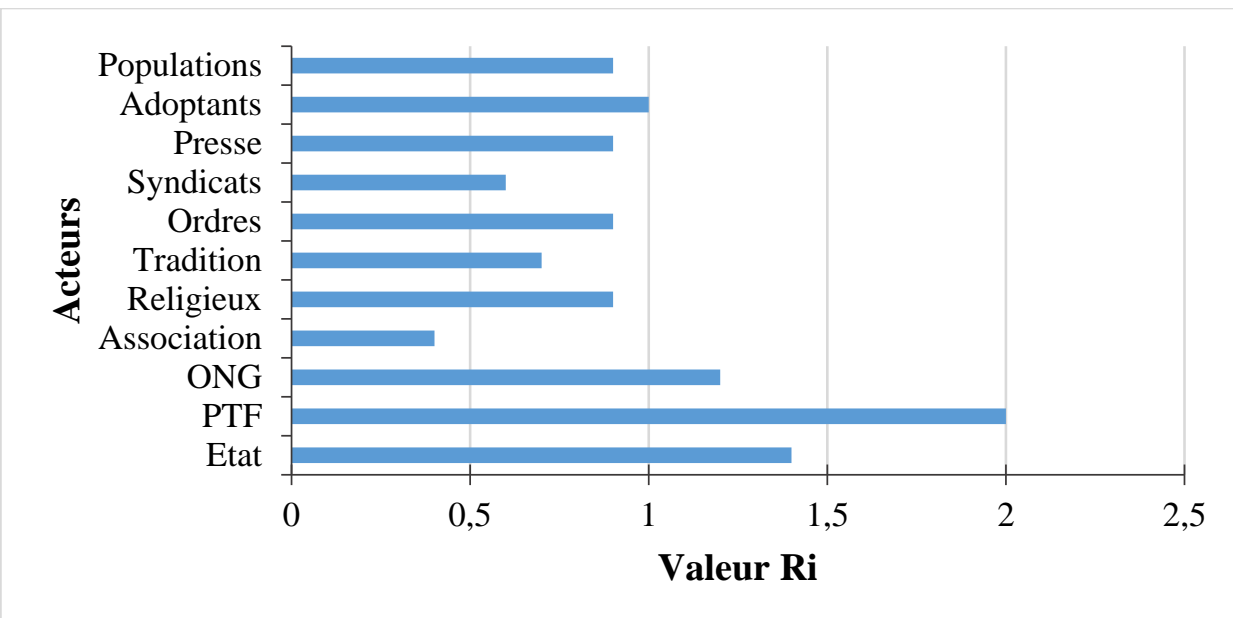

Figure 2 : Rapports de force directs et indirects entre acteurs de la planification familiale

Source : Données de terrain, février-novembre, 2015.

L'analyse de la figure 2 permet d'identifier selon les moyens d'action directs et indirects des acteurs, quatre (4) groupes d'acteurs. Par ordre décroissant, on distingue :

- un acteur disposant d'un rapport de force très élevé : les partenaires techniques et financiers. Il est l'acteur dominant du jeu de la planification familiale au Bénin. Ses décisions et projets sont déterminants pour l'évolution de la prévalence contraceptive au Bénin ;

- un second groupe d'acteurs composé des acteurs relais comme l'Etat, les ONG et les adoptants des méthodes de planification familiale. Ces acteurs disposent d'un rapport de force relativement élevé et sont susceptibles de faire passer leurs décisions dans les négociations qui les concernent ;

- le troisième groupe d'acteurs est composé majoritairement d'acteurs autonomes. Il s'agit des organes de presse, des confessions religieuses et des ordres professionnels, auxquelles viennent s'ajouter les autres catégories de populations (acteurs relais) qui n'ont pas adopté de méthodes de planification familiale. Ces acteurs disposent d'un rapport de force moyennement élevé ;

- le dernier groupe est composé des acteurs disposant des rapports de force les plus faibles. Il s'agit des chefferies traditionnelles, des syndicats, des associations de développement. Ce sont des acteurs qui disposent d'une faible influence et d'une forte dépendance. Les chefferies traditionnelles sont des acteurs dominés dans le jeu de la planification familiale. 
Mais pour confirmer ce classement des acteurs selon les rapports de force, il est nécessaire de calculer les rapports de force potentiels en tenant compte de l'intensité des moyens d'action des acteurs les uns sur les autres.

\section{2-2-3- Rapports de force potentiels entre acteurs de la PF}

L'analyse des rapports de force potentiels (Qi) permet de procéder à une comparaison des poids de chaque acteur, afin de déterminer leurs moyens d'action réels. Elle tient compte de la capacité d'un acteur à influencer l'existence, les missions, les projets ou les processus opératoires d'un autre acteur (tableau III).

Tableau III : Analyse comparée des rapports de force entre acteurs de la PF

\begin{tabular}{|c|c|c|}
\hline Acteurs & $\begin{array}{c}\text { Rapports de force } \\
\text { directs et indirects (Ri) }\end{array}$ & $\begin{array}{c}\text { Rapports de force } \\
\text { potentiels (Qi) }\end{array}$ \\
\hline Etat & 1,4 & 1,3 \\
\hline Partenaires Techniques et Financiers & 2,0 & 1,5 \\
\hline Organisations Non Gouvernementales & 1,2 & 1,3 \\
\hline Associations de développement & 0,4 & 0,8 \\
\hline Confessions religieuses & 0,9 & 1,1 \\
\hline Chefferies traditionnelles & 0,7 & 0,8 \\
\hline Ordres professionnels & 0,9 & 0,7 \\
\hline Syndicats & 0,6 & 0,7 \\
\hline Organisations de Presse & 0,9 & 0,9 \\
\hline Adoptants des méthodes de PF & 1,0 & 1,0 \\
\hline Populations & 0,9 & 0,8 \\
\hline
\end{tabular}

Source : Données de terrain, février-novembre, 2015.

L'analyse du tableau III permet d'observer des sur-classements et des déclassements au niveau des acteurs de la PF. On parle de sur-classement, lorsque le poids de l'acteur connait une augmentation lorsqu'on passe aux rapports de force potentiels (N. Bassaler, 2004. Ainsi, on note une croissance du poids de certains acteurs : les associations de développement (de 0,4 à 0,8$)$, les confessions religieuses (de 0,9 à 1,1 ), les chefferies traditionnelles (de 0,7 à 0,8$)$, les syndicats (de 0,6 à 0,7$)$ et les ONG (de 1,2 à 1,3). Tous ces acteurs gagnent en moyens d'action potentiels, ce qui peut traduire une meilleure intégration sociale de la problématique de la $\mathrm{PF}$, et par ricochet une meilleure implication des segments sociaux.

Par contre, il faut relever le déclassement de l'acteur dominant que constituent les PTF (de 2,0 à 1,5), ainsi que l'Etat (de 1,4 à 1,3), les ordres professionnels (de 0,9 à 0,7$)$, les populations (de 0,9 à 0,8$)$. Le déclassement est la diminution sensible du poids de certains acteurs. Toutefois, les organisations de presse conservent la valeur de leurs moyens d'actions inchangés.

En somme, cette classification des acteurs révèle un jeu complexe et instable des acteurs de la planification familiale au Bénin. L'analyse des rapports de force potentiels montre une concentration des acteurs autour du 
poids moyen $(0,8)$. Les jeux d'alliances basés sur la redéfinition des rôles et l'émergence de projets intégrateurs seront déterminants pour l'évolution du secteur de la planification familiale au Bénin.

\section{2-2-4- Analyse de la connexité entre les acteurs de la planification familiale au Bénin}

Les indicateurs de connexité sont calculés à partir des valeurs de la diagonale figurant dans la matrice des influences directes et indirectes entre acteurs (MIDI). Ils permettent de mesurer pour chaque acteur les pressions qu'il subit et transmet de la part des autres acteurs (F. Azalou, 2015). Au regard des marges de manœuvre des différents acteurs, il s'agit de se poser la question de savoir: "L'acteur peut-il décider seul?». Les indices de connexité $\left(\mathrm{Ci}^{*}\right)$ servent à identifier l'importance des boucles de rétroaction des acteurs sur eux-mêmes et le degré d'imbrication de l'acteur dans la régulation du jeu. L'analyse du degré d'imbrication des acteurs dans le domaine de la planification familiale permet d'observer la configuration illustrer par la figure 3 .

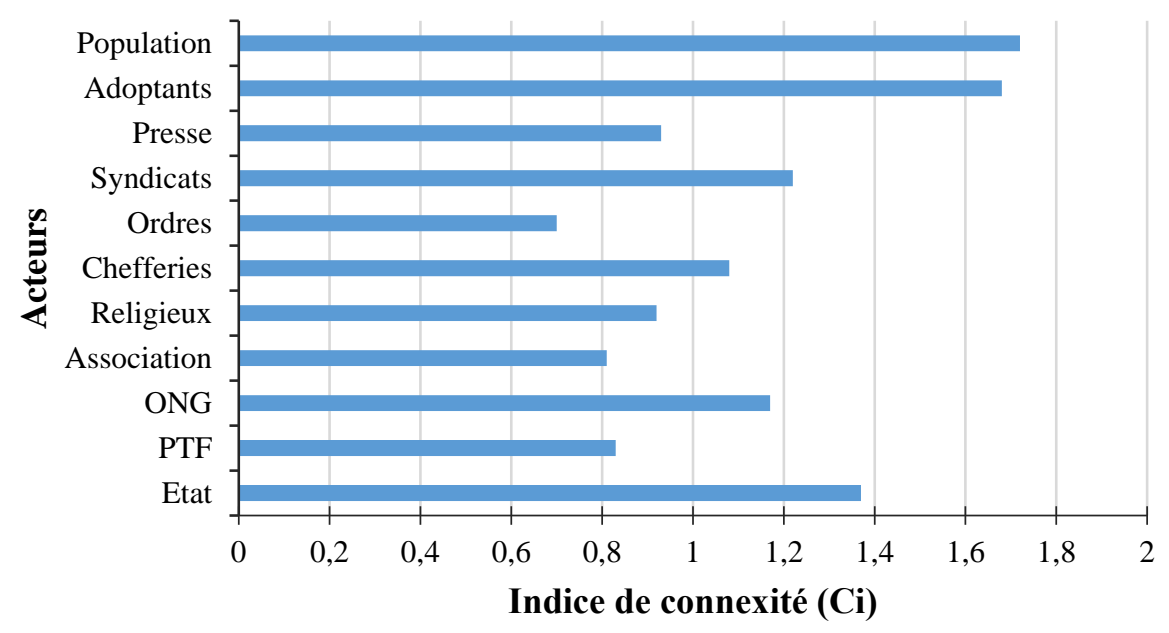

Figure 3 : Connexité actuelle des acteurs de la planification familiale au Bénin Source : Données de terrain, février-novembre, 2015.

L'analyse de la figure 3 permet de distinguer trois groupes d'acteurs :

- Un premier groupe de deux acteurs fortement imbriqués dans le jeu, et dont l'indice de connexité est largement supérieur à la moyenne (égale à 1) : les populations et les adoptants des méthodes de PF. Ces acteurs ne peuvent influencer le jeu qu'avec l'accord des autres acteurs. Toutefois, ils sont les acteurs les plus sollicités et ne disposent pas d'une marge de manœuvre suffisante pour décider et agir seuls. 
- Le deuxième groupe est constitué des acteurs dont l'indice est légèrement supérieur à la moyenne. Il s'agit de l'Etat, des syndicats, des ONG et des chefferies traditionnelles. Avec les acteurs du premier groupe, ils constituent les acteurs clés pour la compréhension du fonctionnement du jeu. C'est en leur sein que les alliances, conflits et évolutions fonctionnelles seraient les plus importantes.

Enfin, on distingue les acteurs dont l'indice de connexité est inférieur à la moyenne. Ce sont les organes de presse, les confessions religieuses, les PTF, les associations de développement et les ordres professionnels. Ces acteurs sont moyennement sollicités dans la régulation du jeu. Il faut noter que la position des PTF dans ce classement semble confirmer les résultats obtenus dans la structure des influences et les rapports de force des acteurs, car ils constituent l'acteur dominant du jeu, disposant également du rapport de force le plus élevé.

\section{2-2-5- Détermination de la marge de manouvre des acteurs de la planification familiale au Bénin}

La détermination de la marge de manœuvre d'un acteur prend en compte la comparaison de son poids et de sa connexité dans le jeu. Cette comparaison est rendue possible par le fait que les rapports de force (poids) et la connexité sont calculés à partir de la matrice des influences directes et indirectes (MIDI). La figure 4 présente la comparaison du poids et de la connexité des acteurs de la planification familiale au Bénin.

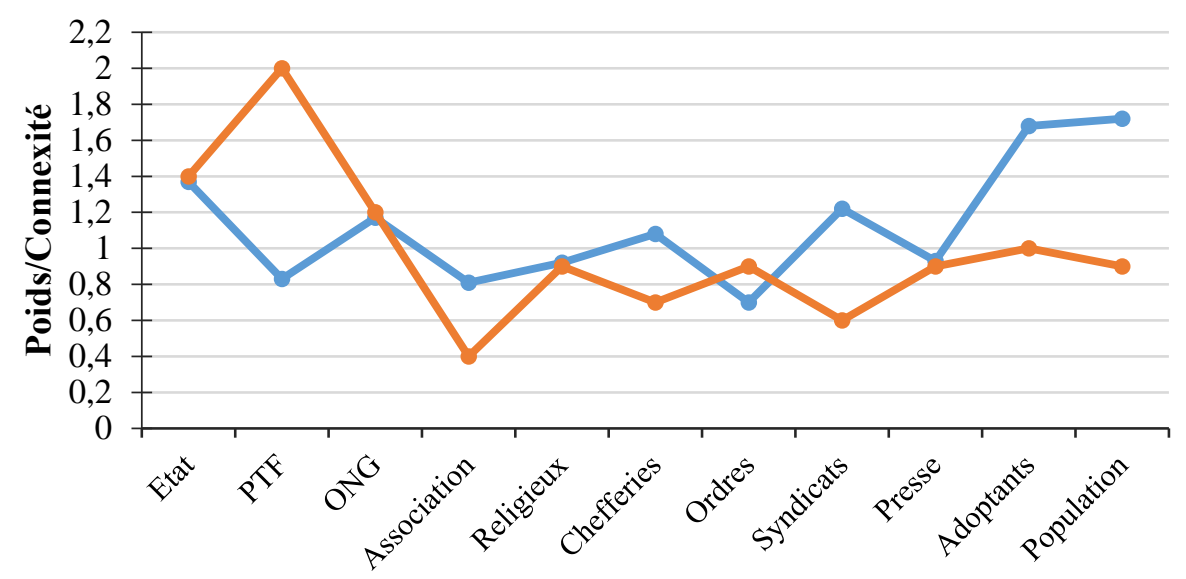

Acteurs

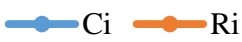

Figure 4 : Comparaison du poids ( $\mathrm{Ri}$ ) et de la connexité $(\mathrm{Ci})$ des acteurs de la PF Source : Données de terrain, février-novembre, 2015. 
L'analyse de la figure 4 permet d'observer trois groupes d'acteurs dans la catégorisation selon la marge de manœuvre.

- Le premier groupe est composé des acteurs disposant d'une marge de manœuvre importante. Il s'agit des partenaires techniques et financiers, acteurs dominants du jeu qui peuvent imposer leurs décisions sans avoir besoin de les négocier. Leur poids est nettement plus élevé que leur connexité, et ils disposent d'une marge de manœuvre suffisante pour orienter les décisions dans le domaine de la planification familiale au Bénin. Il faut signaler que les ordres professionnels ont également un indicateur de rapport de force supérieur à leur connexité. Toutefois, la valeur des deux indicateurs étant inférieure à la moyenne (égale à 1), il dispose d'une marge manœuvre moins importante que les PTF.

- Le deuxième groupe rassemble les acteurs disposant d'une marge de manœuvre relativement réduite. Il s'agit des associations de développement, des chefferies traditionnelles, des syndicats, des adoptants des méthodes de PF et de la population en générale. Dans le jeu, leur connexité est plus élevée que leur rapport de force (poids). Ils sont considérés comme «pris en tenaille» et ne peuvent agir directement. Ces acteurs sont contraints de négocier et de rechercher des alliances avec d'autres acteurs (en l'occurrence les PTF) pour faire passer leurs décisions ou projets.

- L'Etat, les ONG et dans une moindre mesure, les confessions religieuses et les organes de presse constituent le groupe des acteurs dont les indicateurs de rapport de force et la connexité sont de même ordre, et supérieurs à la moyenne (égale à 1). Dans cette configuration, l'Etat et les ONG sont les principaux acteurs susceptibles de prendre part correctement à la régulation du domaine de la planification familiale au Bénin.

Au total, on peut retenir que dans le domaine de la planification familiale au Bénin, la structure des influences des acteurs fait apparaitre des acteurs dominants, des acteurs dominés, des acteurs relais et des acteurs autonomes. De même, l'analyse révèle des rapports de force instables et un domaine généralement très connexe. La plupart des acteurs enregistrent un rapport de force moins élevé que la connexité. L'analyse des positions des acteurs par rapport aux objectifs de la planification au Bénin donne des résultats riches d'enseignements.

\section{2-3- Positionnement des acteurs sur les objectifs de la planification familiale au Bénin}

L'analyse de la position des acteurs face aux objectifs retenus pour la PF au Bénin se base sur la seconde matrice d'entrée du logiciel MACTOR : la 
matrice acteurs/objectifs. Elle permet d'évaluer l'implication des acteurs sur les objectifs, en même temps que les points de convergences et de divergences.

\section{2-3-1- Objectifs de la planification familiale à forte implication des acteurs}

Les objectifs les plus impliquants recouvrent dans l'arène, les principaux champs de bataille sur lesquels les acteurs de la planification familiale s'affrontent. La figure 5 illustre les objectifs qui impliquent le plus les acteurs.

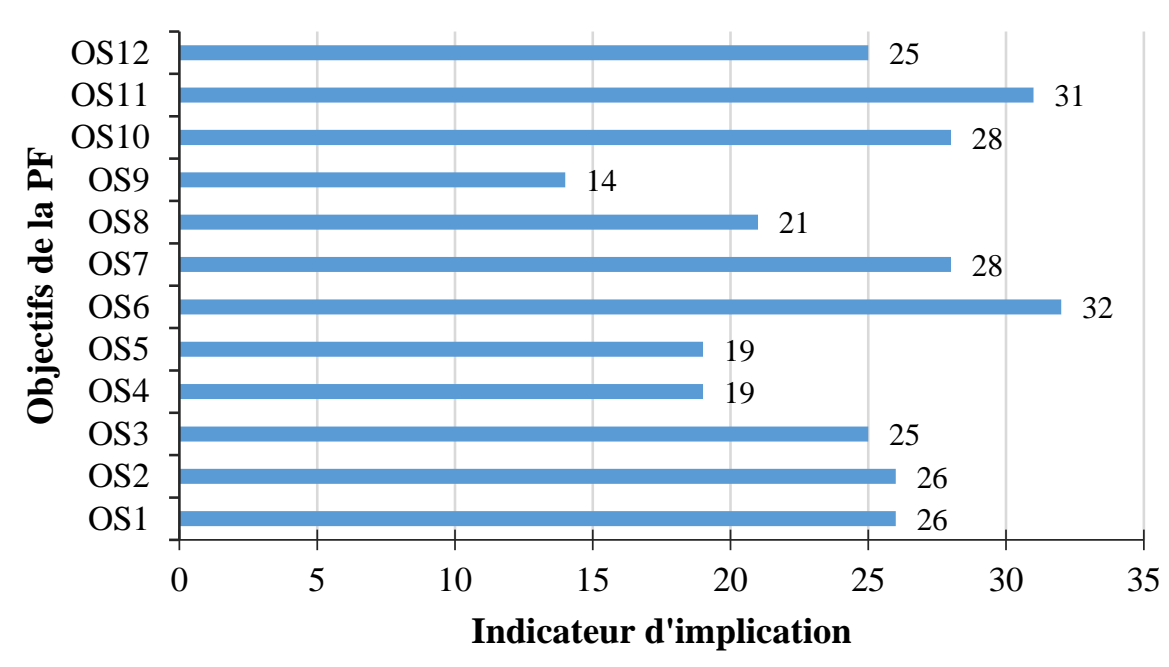

Figure 5 : Objectifs de la planification familiale impliquant le plus d'acteurs au Bénin Source : Données de terrain, février-novembre, 2015.

De l'analyse de la figure 5, on peut retenir comme objectifs à forte implication des acteurs : l'amélioration de l'accessibilité financière de la planification familiale (OS6), la mobilisation des ressources additionnelles pour le repositionnement de la planification familiale (OS11), le financement par le budget de l'Etat de l'approvisionnement en produits de PF (OS10), la vulgarisation et la mise en application des lois sur la santé de reproduction (OS7). Ces objectifs qui impliquent le plus d'acteurs sont pour la plupart en lien avec les ressources financières. L'argent constitue donc le nœud du jeu, le principal champ de bataille où s'affrontent les différents acteurs de la planification familiale au Bénin.

\section{2-3-2- Objectifs de la planification familiale à forte mobilisation des acteurs}

La prise en compte des rapports de force ne modifie pas fondamentalement le classement obtenu pour les objectifs les plus impliquants. La figure 6 présente la hiérarchisation des objectifs suivant le degré de mobilisation. 


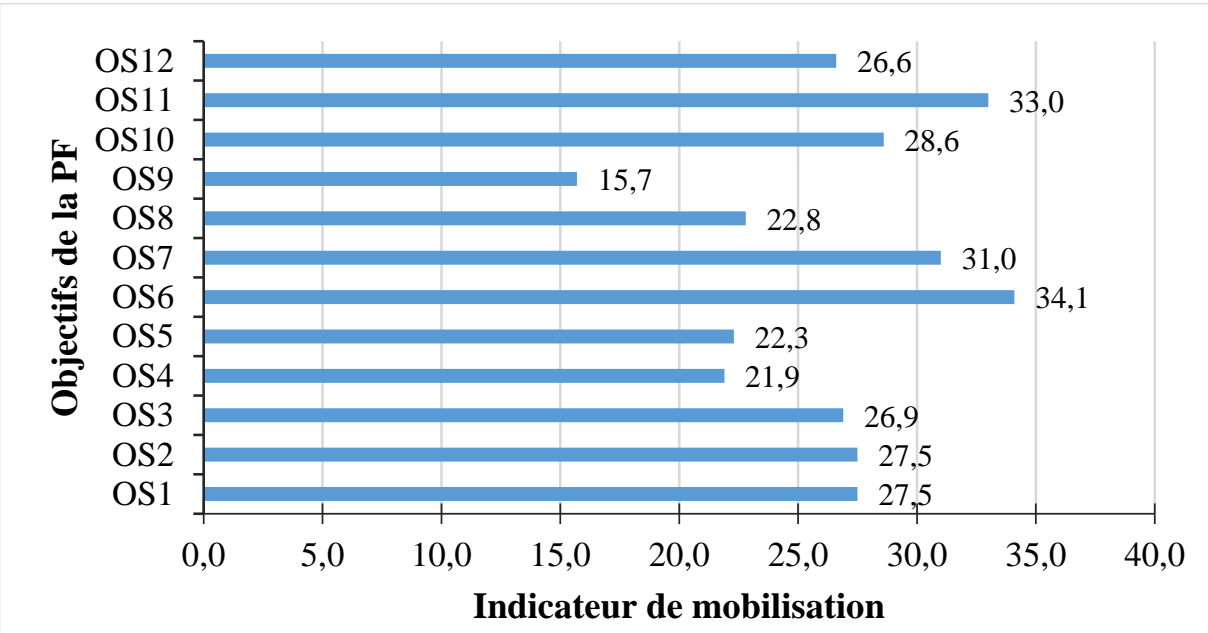

Figure 6 : Objectifs de la planification familiale les plus mobilisateurs des acteurs au Bénin

Source : Données de terrain, février-novembre, 2015.

Ainsi, les objectifs les plus impliquants sont aussi les plus mobilisateurs des acteurs. Le principal déclassement observé concerne le financement par le budget de l'Etat de l'approvisionnement en produits de la planification familiale (OS10). Les autres objectifs qui mobilisent moyennement sont : l'élaboration et la mise en œuvre d'un plan de plaidoyer en direction des décideurs (OS1), d'un plan intégré de mobilisation sociale et de communication pour un changement de comportement (OS2), le renforcement de la capacité des prestataires de soins en sécurisation des produits de la Planification Familiale (OS3) et le renforcement du mécanisme de suivi/évaluation des activités de PF (OS12).

\section{Conclusion}

La planification familiale au Bénin fait appel à une pluralité d'acteurs aux caractéristiques diverses mais agissant vers un même but, l'amélioration de la prévalence contraceptive au Bénin. Les acteurs de la société civile jouent un rôle important dans l'atteinte de cet objectif. Les domaines prioritaires de leurs actions concernent le renforcement de la communication, l'amélioration de l'accès aux services, le renforcement du cadre institutionnel, l'amélioration de l'accessibilité financière à la $\mathrm{PF}$ et le renforcement du cadre de suivi/évaluation. Plusieurs inconvénients sont évoqués pour justifier l'adoption ou non de la contraception. Ainsi, la planification familiale, pour être acceptée et devenir efficace, devra s'intégrer dans le tissu social en prenant en compte les normes socio-culturelles et économiques qui régissent les conditions de la reproduction humaine dans chaque milieu. 


\section{References:}

1. Azalou Tingbé F.V.B. (2015). Gouvernance des changements climatiques face au défi de l'adaptation dans le Centre-Bénin. Thèse de Doctorat Unique, Université d'Abomey-Calavi.

2. Barcelona, D., Bautista, P. et Bogue, D. (1982). La contraception. Guide des méthodes de planning familial, Chicago/Illinois, The Community and Family Study Center.

3. Bassaler N. (2004). Le jeu des acteurs de l'information géographique : un cas appliqué de la méthode MACTOR, Cahier du LIPSOR n ${ }^{\circ} 7$, Paris, CNAM.

4. Delanne P. et Guingnido, J. G. (Dir.) (2003). La santé de la reproduction au Bénin : défis et perspectives, Rapport national sur l'Etat et le devenir de la population du Bénin (REP 2002), Cotonou : MPPD, UNFPA.

5. Godet M. (2001). Manuel de prospective stratégique, Tome 2 : l'art et la méthode, Paris, Dunod.

6. Hountondji T. (2012). Situation socio-économique des ménages: l'adhésion des couples d'intellectuels à la planification familiale dans le $13^{\text {ème }}$ arrondissement/Agla, Mémoire de Maitrise en SociologieAnthropologie, Abomey-Calavi : DSA/FLASH/UAC.

7. Kuyu C., Kéita D. (2011). Les barrières sociales et culturelles à la généralisation de la planification familiale et à une meilleure santé maternelle au Bénin, UNFPA-Bénin, Cotonou, Espérance.

8. Wallace E. et Goundété E., (1996). Revue documentaire en santé reproductive et planification familiale $(S R / P F)$ au Bénin, Cotonou : MS/DNPS, UNFPA. 